\title{
In vivo tmRNA protection by SmpB and pre-ribosome binding conformation in solution
}

\author{
EHSAN RANAEI-SIADAT, ${ }^{1,2,8}$ CÉCILE MÉRIGOUX, ${ }^{3,4,8}$ BILI SEIJO, ${ }^{1,2}$ LUC PONCHON, ${ }^{1,2}$ JEAN-MICHEL SALIOU, ${ }^{5,9}$ \\ JULIE BERNAUER, ${ }^{6,7}$ SARAH SANGLIER-CIANFÉRANI, ${ }^{5}$ FRÉDERIC DARDEL, ${ }^{1,2}$ PATRICE VACHETTE, ${ }^{3,4}$ \\ and SYLVIE NONIN-LECOMTE, \\ ${ }^{1}$ CNRS-UMR 8015, Laboratoire de Cristallographie et RMN Biologiques, Faculté de Pharmacie, 75270 Paris Cedex 06, France \\ ${ }^{2}$ Université Paris Descartes, LCRB, Faculté de Pharmacie, 75270 Paris Cedex 06, France \\ ${ }^{3}$ Université Paris-Sud, IBBMC, UMR8619, 91405 Orsay, France \\ ${ }^{4} \mathrm{CNRS}, 91405$ Orsay, France \\ ${ }^{5}$ CNRS, IPHC-LSMBO, Université Louis Pasteur Bât, 67087 Strasbourg, France \\ ${ }^{6}$ AMIB, INRIA Saclay-île de France, 91120 Palaiseau, France \\ ${ }^{7}$ LIX, CNRS UMR 7161, École Polytechnique, 91120 Palaiseau, France
}

\begin{abstract}
TmRNA is an abundant RNA in bacteria with tRNA and mRNA features. It is specialized in trans-translation, a translation rescuing system. We demonstrate that its partner protein SmpB binds the tRNA-like region (TLD) in vivo and chaperones the fold of the TLD-H2 region. We use an original approach combining the observation of tmRNA degradation pathways in a heterologous system, the analysis of the tmRNA digests by MS and NMR, and co-overproduction assays of tmRNA and SmpB. We study the conformation in solution of tmRNA alone or in complex with one SmpB before ribosome binding using SAXS. Our data show that $\mathrm{Mg}^{2+}$ drives compaction of the RNA structure and that, in the absence of $\mathrm{Mg}^{2+}, \mathrm{SmpB}$ has a similar effect albeit to a lesser extent. Our results show that tmRNA is intrinsically structured in solution with identical topology to that observed on complexes on ribosomes which should facilitate its subsequent recruitment by the 705 ribosome, free or preloaded with one SmpB molecule.
\end{abstract}

Keywords: tmRNA; SmpB; trans-translation; in vivo protection; SAXS; NMR

\section{INTRODUCTION}

With its protein partner SmpB, tmRNA is the major actor of bacterial trans-translation, a quality control mechanism of translation and a ribosome rescuing system. As inferred from its name, tmRNA acts both as a tRNA and an mRNA. Its length is $2350 \mathrm{nt}$, depending on bacteria. Computational studies show that its tertiary structure is a necklace of several structural blocks (1 MLD and 4 PKs) attached by a linker helix (H2) to a tRNA-like domain (TLD) formed by the pairing of the $5^{\prime}$ and the $3^{\prime}$ ends (Fig. 1) and that the occurrence of each block is extremely well conserved (Burks et al. 2005).

Trans-translation is a multistep process involving tmRNA and SmpB binding to the ribosome. In the early life of tmRNA in the cell, the TLD is charged by the alanyl-tRNAsynthetase (Komine et al. 1994). This mandatory aminoacylation reaction is enhanced by the presence of SmpB (Hanawa-

\footnotetext{
${ }^{8}$ These authors contributed equally to this work.

${ }^{9}$ Present address: Institut Pasteur de Lille, 59019 Lille Cedex, France Corresponding authors: sylvie.nonin@parisdescartes.fr, Patrice.Vachette@u-psud.fr

Article published online ahead of print. Article and publication date are at http://www.rnajournal.org/cgi/doi/10.1261/rna.045674.114.
}

Suetsugu et al. 2002; Shimizu and Ueda 2002), which suggests that SmpB modulates the TLD structure to enhance recognition by the synthetase. Apart from the prebinding TLDalanylation step, efforts have been focused on ribosome recognition and on the subsequent steps such as ribosome decoding center hijacking (Nonin-Lecomte et al. 2009; Neubauer et al. 2012). Cryo-EM has provided beautiful and extremely informative pictures of how the [tmRNA.SmpB] complex performs its task, visualizing various stages from pre-accommodation to post-translocation (Valle et al. 2003; Kaur et al. 2006; Cheng et al. 2010; Fu et al. 2010; Weis et al. 2010). Comparatively few papers address the prebinding $3 \mathrm{D}$ structure of tmRNA. NMR and X-ray structures of the TLDs of Aquifex aeolicus and Thermus thermophilus confirm the RNA L-shape structure of the TLD (Gaudin et al. 2003; Gutmann et al. 2003; Bessho et al. 2007; Ramrath et al. 2012) and the formation of a very stable pseudoknot formed

(C) 2014 Ranaei-Siadat et al. This article is distributed exclusively by the RNA Society for the first 12 months after the full-issue publication date (see http:// rnajournal.cshlp.org/site/misc/terms.xhtml). After 12 months, it is available under a Creative Commons License (Attribution-NonCommercial 4.0 International), as described at http://creativecommons.org/licenses/by-nc/4.0/. 
A

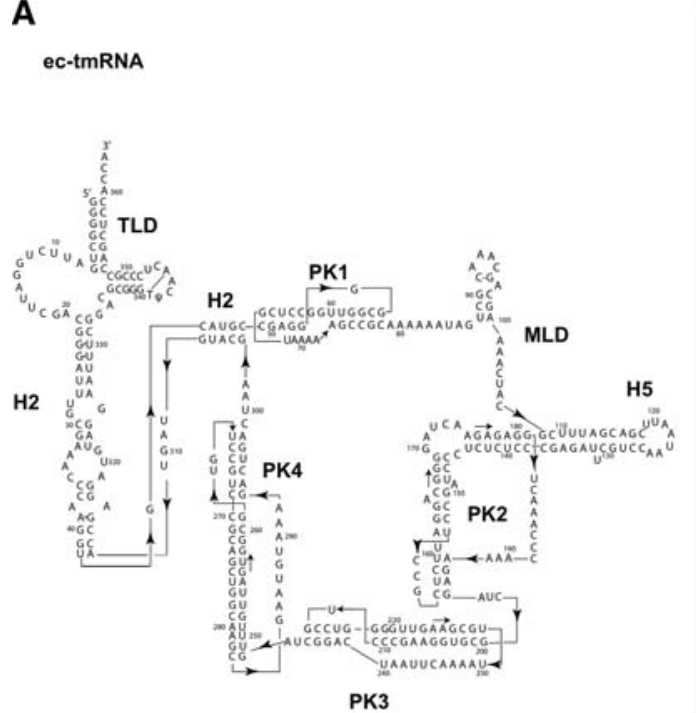

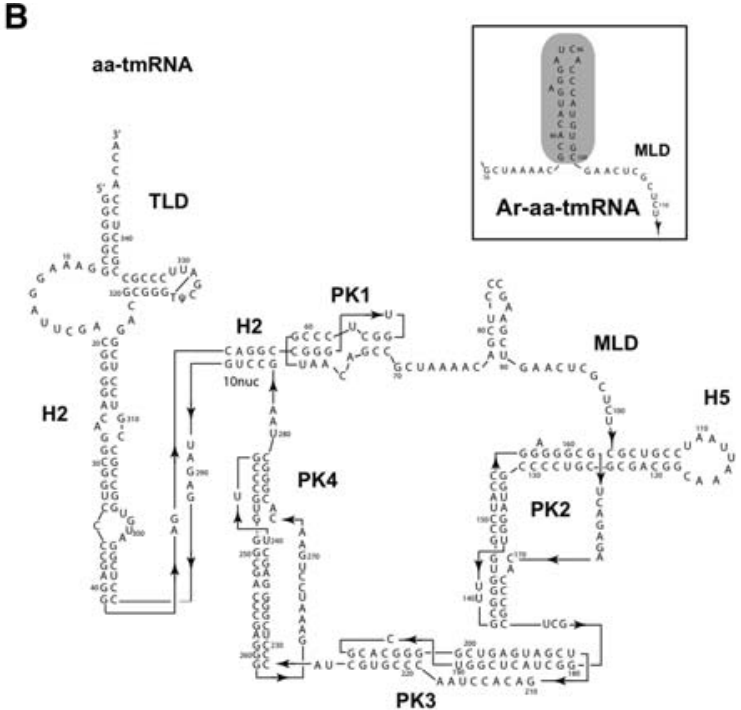

FIGURE 1. Secondary structure models of $(A)$ ec-tmRNA and $(B)$ aa-tmRNA. (Inset) Modified MLD of the armored aa-tmRNA with the MS2 encapsidation stem-loop shaded in gray.

by the PK1 sequence (Nonin-Lecomte et al. 2006). Three-dimensional models were built by Burks et al. (2005). Cryo-EM structures of partial or full-length tmRNAs bound to the ribosome confirm the suspected flexibility of the global conformation, with a 3D structure articulated around the different structural blocks to go through the various stages of its task. However, no experimental information on the three-dimensional structure of the full-length molecule before ribosome binding is available yet.

In this paper, we address the question of whether the $3 \mathrm{D}$ structure of tmRNA is preorganized for ribosome binding and to what extent the binding of the first SmpB molecule will modulate this structure. Taken together, biochemistry and structural studies have evidenced three potential SmpB binding sites on tmRNA. Wower et al. (2002) showed that up to three $\mathrm{SmpB}$ molecules can crosslink with a large portion of the TLD-H2 block of E. coli tmRNA. The cryo-EM structure of Kaur et al. (2006) led the authors to conclude that two molecules of SmpB can bind the TLD. According to chemical and enzymatic probing experiments, $\mathrm{SmpB}$ can also establish contacts with the tmRNA large loop in vitro, in particular with PK1 and PK4 (Metzinger et al. 2005). This third binding site would be the site of highest affinity with a $K_{\mathrm{d}}$ of $\sim 3 \mathrm{nM}$ vs. 10 for the TLD (Metzinger et al. 2008). The present study is focused on the binding of the first molecule of SmpB. The localization of the binding site is probed in vivo by original co-production assays of heterologous tmRNAs and proteins. Three tmRNAs were overproduced in Escherichia coli (Fig. 1), the endogenous E. coli tmRNA (ec-tmRNA), the heterologous A. aeolicus (aatmRNA), and one chimeric derivative (ec-tmRNA-aaMLD). TmRNA and SmpB were both produced in E. coli and purified in native conditions. The tmRNA conformation in solution before ribosome binding was probed by small angle X-ray scattering (SAXS). From our experimental data, we could derive models for the conformation of full-length tmRNA. We also demonstrate that the binding of a single SmpB molecule can drive a compaction of the whole RNA structure, an effect similar to that of high magnesium concentration.

\section{RESULTS}

\section{Overproduction of homologous and heterologous tmRNA in E. coli}

The production of full-length ec-tmRNA in E. coli cells was reported in the literature (Felden et al. 1997). We have cloned the genes of the 363-nt ec-tmRNA (ec-ssrA) and of the heterologous A. aeolicus 347-nt aa-tmRNA (aa-ssrA) in pBSTNAV plasmids (Meinnel et al. 1998). Each plasmid was used to transform JM101tr cells. This plasmid-strain association allows high levels of RNAs to be transcribed under the control of the constitutive lpp promoter and yields high amounts of processed full-length ec-tmRNA (Fig. 2A, lane 5). A. aeolicus is a hyperthermophile bacterium whose optimal growth temperature is $\sim 90^{\circ} \mathrm{C}$ (Deckert et al. 1998). Its tmRNA is thus expected to be highly resistant at $37^{\circ} \mathrm{C}$. However, despite the existence of several highly structured elements known to exhibit high-resistance to RNase degradation (TLD, PK1-4), the gel of the total RNA extracts reveals that aa-tmRNA undergoes discrete and systematic degradation in E. coli (Fig. $2 \mathrm{~A}$, lane 3). It exhibits one faint band (F) corresponding to the expected full-length aa-tmRNA and two strong bands (D1 and D2) at lower than expected molecular weights (the in vitro transcript of the aa-tmRNA was loaded on the same gel as a migration marker). The RNA digests evidenced by 


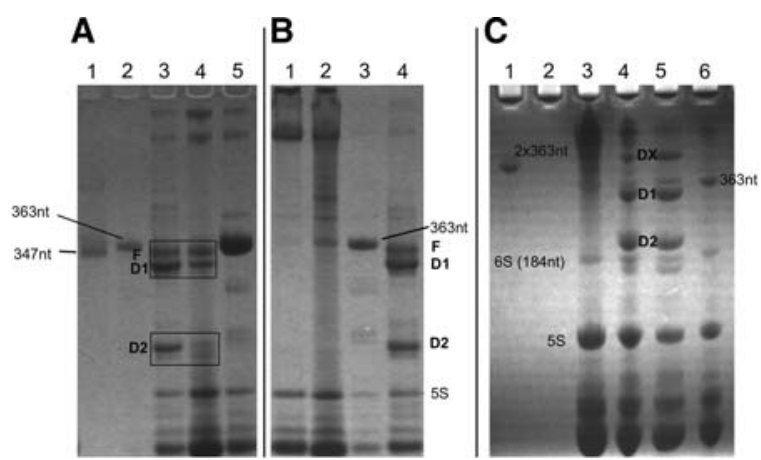

FIGURE 2. $(A, B) 4 \%-12 \%$ Bis Tris PAGE. (A) Lanes 1,2: Markers-( 1 ) purified aa-tmRNA (in vitro transcript), (2) purified recombinant ectmRNA. Lanes 3-5: Total RNA extracts for overproduction of recombinant tmRNAs- $(3,4)$ total RNA extracts from overproduction of aatmRNA, respectively, in the absence and in the presence of SmpB, (5) overproduction of ec-tmRNA. (B) Total RNA extracts from ectmRNA-aa-MLD at two different concentrations (lanes 1,2), from ectmRNA (lane 3), and aa-tmRNA (lane 4). (C) $8 \mathrm{M}$ urea 12\% PAGE. Purified aa-tmRNA transcribed in vitro migrating as a multimer, probably a dimer (lane 1). Lanes 3-6: Total RNA extracts from JM101tr cells transformed with empty pBSTNAV (lane 3), aa-ssrA-pBSTNAV (lanes 4,5 ), and ec-ssrA-pBSTNAV (lane 6). Bands Dx in lanes 4 and 5 correspond to an oligomeric species and have no equivalent in lane 6.

bands D1 and D2 are hereafter named aa-tmRNA-D1 and aatmRNA-D2, respectively (D standing for digest). The comparison of Bis Tris (Fig. 2A) and 8M acryl-urea (Fig. 2C) gels of total RNA extracts shows that full-length aa-tmRNA transcribed either in vivo or in vitro forms dimers and/or aggregates. The intermolecular interactions between RNA strands responsible for this effect are strong enough to withstand $8 \mathrm{M}$-urea but are prevented by SDS, the negative charges of which repel RNA strands. According to the gels, the size of aa-tmRNA-D2 is $\sim 200 \mathrm{nt}$, which is consistent with a digest encompassing the PK4-H5 region. We have overproduced a 196-nt RNA recapitulating aa-tmRNA residues 97-292 (Fig. 3A, below), i.e., the PK4-H5 block framed by a few flanking nucleotides. The gel of the RNA extract shows evidence of degradation similar to that observed with full-length aatmRNA, with a single species migrating exactly at the same position as aa-tmRNA-D2. This shows that the necklace of pseudoknots and the neighboring helix $\mathrm{H} 5$ act as degradation stoppers. The gels also exhibit a faint band at a lower molecular mass, which could arise from further digestion of the flanking nucleotides and $\mathrm{H} 5$ helix.

Recombinant ec-tmRNAs and aa-tmRNA-D1 were purified using the three-step protocol described in the Materials and Methods section. Their respective molecular mass (MM) was determined in native conditions by supra-molecular TOF/mass spectrometry. Ec-tmRNA MM is $117 \mathrm{kDa}$ as expected (Supplemental Fig. 1). Aa-tmRNA-D1 actually corresponds to a mix of two digests that are very close in mass $(104,396.7 \pm 28.9 \mathrm{Da}$ and $104,702.8 \pm 10.6 \mathrm{Da})$ and probably differ by a single nucleotide. The mass default ( $\sim 7.5$ and $7.8 \mathrm{kDa}$ ) compared to the expected full-length aa-tmRNA approximately corresponds to $25 \mathrm{nt}$. The two species are not resolved on the gels of RNA extracts, and for simplification they will be hereafter considered as one single species (D1). The denaturing ionization of purified species D1 reveals the presence of a single strand with a MM of $80,650 \pm$ $20 \mathrm{Da}$ corresponding to $\sim 250 \mathrm{nt}$. Taken together, these results show that, despite its migration as a single band on gels, aa-tmRNA-D1 is a mixture of double-stranded species.

\section{Band shifts experiments}

The experiment illustrated in Figure 3A shows that aatmRNA digestion stops at the PK4-H5 block. Together with the mass spectroscopy results, it suggests that in vivo deletion occurs in the MLD. In order to assess the presence

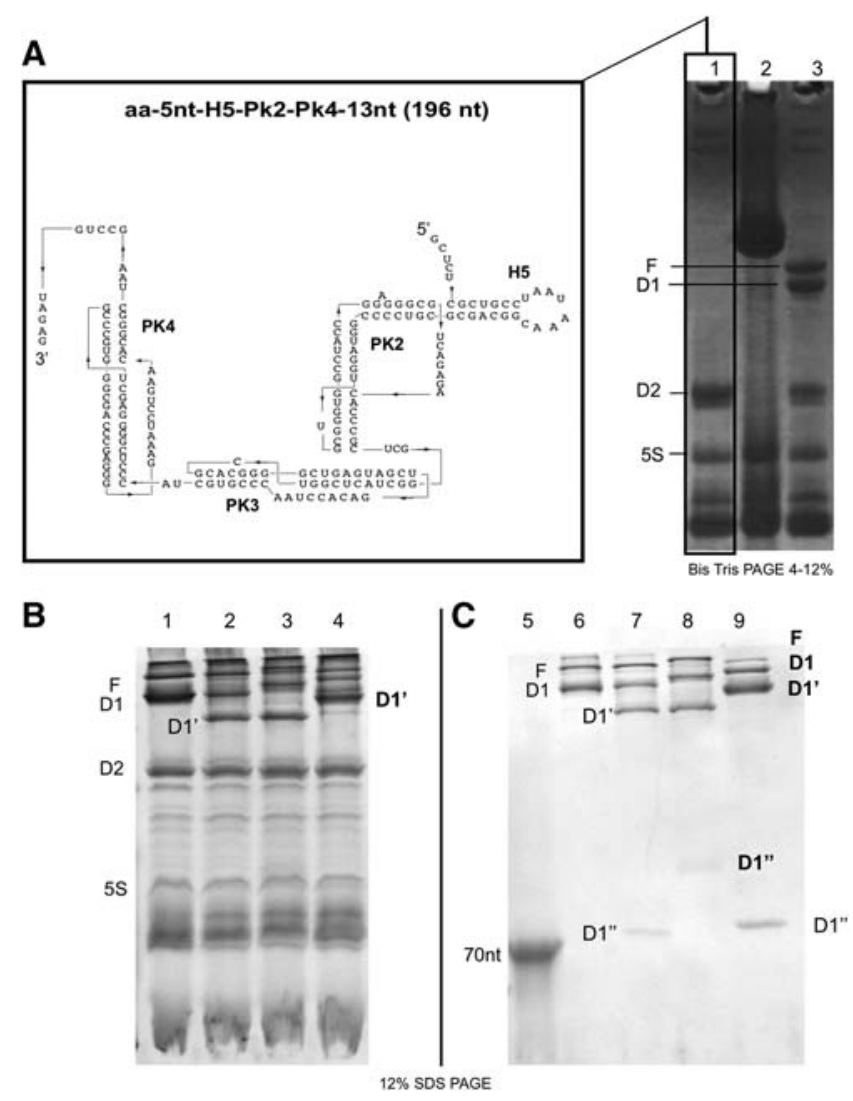

FIGURE 3. (A) (Left) Secondary structure model of the 196-nt RNA encompassing the aa-H5-PK4 region. The corresponding gene (196RNA) was inserted in the pBSTNAV plasmid. (Right) Bis Tris 4\%$12 \%$ PAGE (NuPAGE Novex). Total RNA extracts from JM101tr cells transformed with 196-RNA-pBSTNAV (lane 1), ec-ssrA-pBSTNAV (lane 2), and aa-ssrA-pBSTNAV (lane 3). Lane 1 exhibits a strong band similar to band D2 of lane 3. $(B, C)$ Hybridization experiments on full-RNA extracts from aa-tmRNA overproduction in JM101tr cells $(B)$ and on a partially purified mixture of full-length aa-tmRNA and aatmRNA-D1 $(C)$. Lane 1: extracts loaded at room temperature; lanes 2-4: extracts loaded after a heat-cool cycle, alone (lane 2) and in the presence of antiH2PK1 and antiTLD (respectively, lanes 3 and 4); lane 5: RNA marker of $70 \mathrm{nt}$; lanes 6-9: same conditions, respectively, as lanes 1-4 but on the purified mixture. 
of the TLD and of the H2-PK1 regions, we conducted hybridization experiments that we monitored by band shift observations on $12 \%$ SDS PAGE gels (Fig. 3B,C). Two DNA oligonucleotides, anti-H2PK1 (15 nt, designed to hybridize nucleotides 39-54 of Fig. 1B) and anti-TLD (35 nt, designed to hybridize nucleotides 329347 of Fig. 1B), were used as probes. The experiments were conducted either on RNA extracts (Fig. 3B) or on a partially purified mixture of full-length aatmRNA and aa-tmRNA-D1 (Fig. 3C). Upon heating, the intensity of band D1 disappears, while that of bands $\mathrm{D1}^{\prime}$ and $\mathrm{D1}^{\prime \prime}$ increases (lanes 2 and 7). Migration of band D1 matches the 250-nt species observed at $\sim 80 \mathrm{kDa}$ in $\mathrm{MS}$ spectroscopy. Band $\mathrm{D1}^{\prime \prime}$ is consistent with the 75-nt species expected after MLD digestion and corresponding to the $5^{\prime}$ strand of the

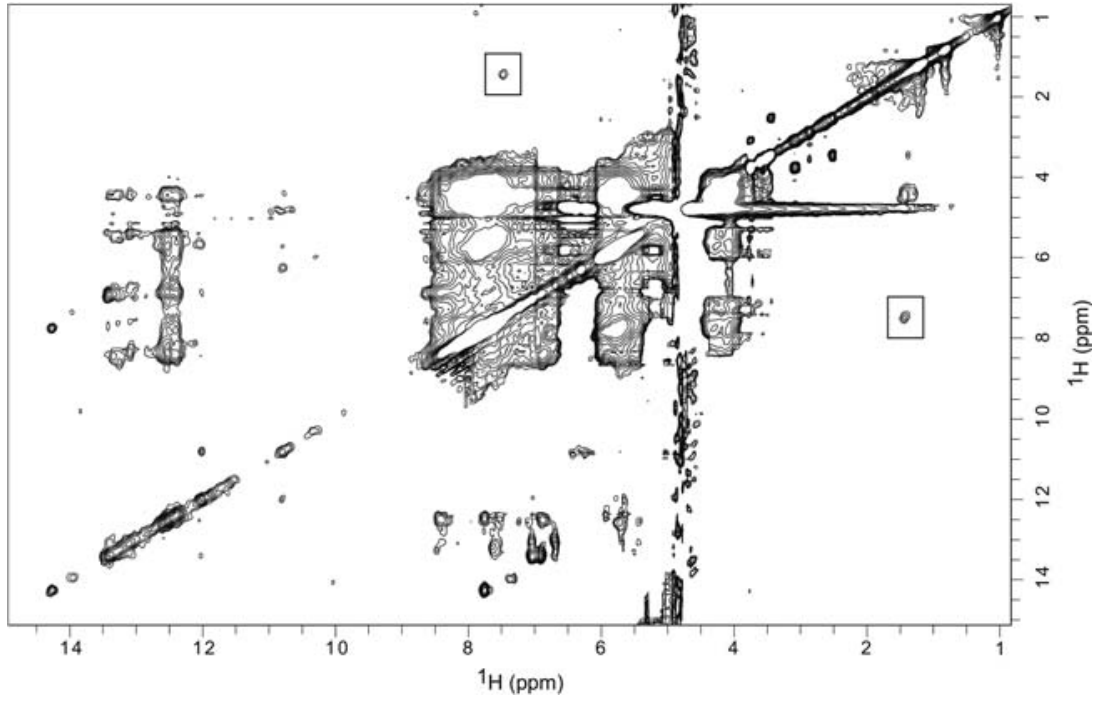

FIGURE 4. 2D NOESY-Watergate NMR spectrum of aa-tmRNA-D1 purified in native conditions, recorded in $95 \% \mathrm{H}_{2} \mathrm{O}-5 \% \mathrm{D}_{2} \mathrm{O}$ at $293 \mathrm{~K}$ and $\mathrm{pH}$ 6.5. The exchangeable proton region (between 9.5 and $14.5 \mathrm{ppm}$ ) shows that at least part of the molecule is folded. The boxed H6-methyl cross-peaks reveal the presence of the $3^{\prime}$ side of the full-length aa-tmRNA sequence.

\section{TLD-H2-PK1 region of aa-tmRNA. Hy-}

bridization with anti-TLD shifts bands

F, D1, and D1' (lanes 4 and 9) but not D2 and D1" as expected. This shows that the TLD is retained in aa-tmRNA-D1. Hybridization with anti-H2PK1 shifts bands F, D1, and D1" (lanes 3 and 8 ) but not $\mathrm{D} 1^{\prime}$ and $\mathrm{D} 2$. This shows that the D1 and its shorter component are cleaved in the MLD region, after at least half of the PK1 sequence. Altogether, this also shows that the deletion of $25 \mathrm{nt}$ occurs between PK1 and H5.

\section{NMR analysis of aa-tmRNA-D1}

The TLD block results from the association of the $3^{\prime}$ and $5^{\prime}$ ends (Fig. 1) and its folding topology has much in common with tRNAs (12-15). The previous hybridization experiment shows that aa-tmRNA-D1 retains the 3 '-side of the acceptor arm of the TLD. The TLD undergoes a single post-transcrip-

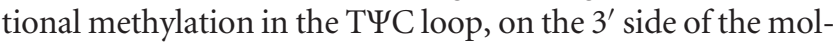
ecule (Felden et al. 1998). We checked for its presence by NMR. The presence of a methyl group transcribes into methyl-imino and methyl-H6 cross-peaks in 2D NOESY spectra, and we recently showed that it is possible to observe such cross-peaks with ec-tmRNA (Ranaei-Siadat et al. 2013). The 2D NOESY spectrum of the purified aa-tmRNA-D1 species at $\sim 104 \mathrm{kDa}$ recorded in $\mathrm{H}_{2} \mathrm{O}$ at $293 \mathrm{~K}$ and $\mathrm{pH} 6.5$ is quite well resolved in the exchangeable proton region (9.5-14.5 ppm) (Fig. 4). Many resonances are missing because they undergo high relaxation rates. Nevertheless, the cross-peak pattern in this region reveals the existence of several WatsonCrick G.C base pairs attesting to the formation of a stable 3D structure. The region of aromatic proton resonances (between 6.5 and $8.5 \mathrm{ppm}$ ) displays the strong and single crosspeak at 7.47 and $1.45 \mathrm{ppm}$, as similarly observed in the aaTLD (Gaudin et al. 2003) and the ec-tmRNA (Ranaei-
Siadat et al. 2013) NOESY spectra. The presence of this methyl resonance demonstrates the presence of the TLD T-arm and T-loop in aa-tmRNA-D1, and thus of the $3^{\prime}$ end nucleotides of full-length aa-tmRNA. Considering the high resistance of tRNAs to RNases and our ability to overproduce the aa-TLD alone using the same plasmid and E. coli strain (Gaudin et al. 2003), we can conclude that aa-tmRNA-D1 contains the full TLD part. The observed mass default thus originates from an endonucleolytic digestion and is consistent with the deletion of the MLD region, and in turn, the longer strand of aa-tmRNA-D1 species would correspond to the $3^{\prime}$ end part of aa-tmRNA (H5-3' end region).

\section{aa-MLD ec-tmRNA chimera}

To probe the weakness of the MLD, the region coding for the ec-MLD (residues 79-108) was replaced in ec-ssr $A$ by the corresponding aa-MLD sequences (residues 70-101) with the view to recreate a substrate for degradation. JM101tr bacteria were transformed with the ec-ssrAaaMLD-pBSTNAV plasmid. The pattern of the corresponding total RNA extracts (Fig. 2B, lanes 1,2) reveals that the MLD substitution entirely destabilizes the ec-tmRNA. The faint band at $363 \mathrm{nt}$ observed in the overloaded lane most probably corresponds to the endogenous production of wild-type ec-tmRNA. Modifications of the MLD were used to engineer functional trans-translational tests (Tanner et al. 2006) and were shown to be compatible with the integrity of tmRNA. In light of this result, the complete degradation of the aa-MLD ec-tmRNA chimera is totally unexpected. However, it brings evidence that the aaMLD is a target for degradation in E. coli as inferred from the aforementioned MS and NMR analyses of aa-tmRNA-D1. 


\section{NMR analysis of the MLD}

Aa-tmRNA-D1 is a by-product of the endonucleolytic degradation of aa-tmRNA. Because tRNA-like structures and pseudoknots are highly resistant, we suspected the aa-tmRNA initial degradation to take place within its least folded part, around the MLD. In 2D models based on chemical and enzymatic probing experiments, the MLD region appears as a stem-loop framed by unstructured single strands. The $E$. coli MLD (ec-MLD) contains a stem-loop of 4 bp (Felden et al. 1997) and that of A. aeolicus (aa-MLD) is a little more structured, with 6 bp (Metzinger et al. 2005). We probed by NMR the structures in solution of 22-mer and 29-mer oligonucleotides recapitulating aa- and ec- MLDs (respectively, 5'-rAGCUCCCGAAGCUGAACUCGCU and 5'-rAAAAAA UAGUCGCAAACGACGAAAACUAC).

The $1 \mathrm{D}$ and $2 \mathrm{D}-\mathrm{NOESY}{ }^{1} \mathrm{H}$ spectra recorded in water are consistent with MFOLD structure predictions (Zuker 2003) and confirm the poor structuring of the MLD from the two species, both of which adopt small stem-loop structures (Supplemental Figs. 2, 3). In particular, the aa-MLD contains three G.C and two A.U Watson-Crick base pairs. It is less structured than modeled from the probing experiments.

\section{Co-overproduction of tmRNA with either SmpB or His6-MS2 coat protein}

In a homologous system (ec-SmpB and ec-tmRNA in E. coli cells), SmpB enhances tmRNA lifetime and stability in the cells and allows recovery of higher tmRNA amounts than when the RNA is produced alone (Hanawa-Suetsugu et al. 2002; Hong et al. 2005). With the idea that SmpB may protect aa-tmRNA from degradation, we decided to co-overproduce the A. aeolicus SmpB (aa-SmpB) and aa-tmRNA in LB medium. Both genes were cloned in our homemade plasmid pProRNA (Ponchon et al. 2013). RNA transcription is constitutively controlled by the $l p p$ promoter, while that of $S \mathrm{mpB}$ is controlled by the inducible lac promoter. The cells were grown in LB growth medium. Digest D2 disappears from the total RNA extracts (Fig. 2A, lane 4). When production is carried out in rich growth medium (2XTY), a faint band reappears at position $\mathrm{D} 2$, probably because the relative proportion of the aa-tmRNA transcript is increased compared to that of SmpB (data not shown). This shows that SmpB blocks the degradation pathway(s) leading to aa-tmRNA-D2 and that the disappearance of band D2 is not a consequence of the inactivation by $\mathrm{SmpB}$ of the RNase(s) involved in the degradation pathway. The presence of band D1 shows that aa-SmpB does not fully protect aa-tmRNA from endonucleolytic degradation.

We recently reported the co-overproduction of the armored version of aa-tmRNA (Ar-aa-mRNA) with the MS2His 6 coat protein (138 aa) using the homemade $\mathrm{p} 44 \mathrm{~K}$ plasmid (Ponchon et al. 2013). The Ar-aa-MLD was modified compared to wild type to incorporate, instead of the short stem- loop of the aa-MLD, the 23-nt-long encapsidation signal stem-loop of the MS2 coat protein (Fig. 1B, inset). This replacement does not protect the MLD from degradation (Fig. 5). The His6-MS2 coat protein binds the RNA as a dimer and, contrary to aa-SmpB, totally blocks the degradation pathway D1 of Ar-aa-tmRNA (Fig. 5, lanes 4-6). As expected, the His6MS2 coat protein does not block the degradation pathway D1 of aa-tmRNA, which lacks the encapsidation signal (Fig. 5, lane 3). If SmpB were bound to the large loop close to the MLD, we would expect a gel pattern close to that observed with the MS2 coat protein. The comparison of the two coproduction experiments thus led us to the conclusion that, when co-overproduced in vivo with aa-tmRNA, aa-SmpB preferentially binds to the TLD.

\section{Structural investigations of ec-tmRNA and ec-tmRNA complexes using SEC-SAXS}

We aimed at obtaining structural data on tmRNA in solution before ribosome binding in the presence or absence of SmpB. The SmpB sequence is extremely conserved across species (Dong et al. 2002; Someya et al. 2003), and this translates into the fact that a heterologous $\mathrm{SmpB}$ can efficiently be active and complement the deletion of the gene of the endogen protein (Thibonnier et al. 2010). In vitro, the heterologous system can perform trans-translation, albeit less efficiently (Ito et al. 2002). Wower et al. (2002) have shown that A. aeolicus SmpB can bind in vitro full-length and truncated ec-tmRNA. As explained in Materials and Methods, our choice finally turned to the heterologous system composed of ec-tmRNA and aaSmpB because atomic coordinates were available for both molecules (Dong et al. 2002; Ramrath et al. 2012). Data were recorded on tmRNA samples alone and in the presence of one molar equivalent of $\mathrm{SmpB}$ protein, both in the presence of $5 \mathrm{mM} \mathrm{Mg}^{2+}$ and in its absence. All samples were loaded onto the on-line SE-HPLC column, and SAXS patterns were recorded along the elution profile. All profiles exhibited a

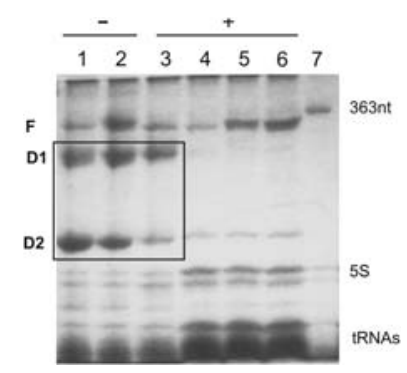

FIGURE 5. His6-MS2 coat protein protects MLD from degradation in vivo. Total RNA extracts loaded onto a 10\% SDS PAGE (UV shadowing). All RNAs were produced in p44K. (-) No protein coproduction, (+) His6-MS2 coat protein production. Lane 1: aa-tmRNA; lane 2: Ar-aa-tmRNA; lane 3: as a control, aa-tmRNA coproduced with the His6-MS2 coat protein; lanes 4-6: Ar-aa-tmRNA coproduced with the His6-MS2 coat protein; three clones tested. The degradation pathway leading to D1 was suppressed by the coproduction of the protein; lane 7: partially purified ec-tmRNA as marker. 
minor fraction of oligomer and a major monomer peak. Scattering patterns of tmRNA alone or in complex with SmpB are shown in Figure 6, in panel A1 without $\mathrm{Mg}^{2+}$ and in panel A2 with $\mathrm{Mg}^{2+}$. Structural parameters derived from each curve are shown in Table 1. Guinier plots are shown in insets to Figure 6, panels A1 and A2, while $p(r)$ functions, which represent histograms of intra-particle distances, are presented in Figure 6, panels $\mathrm{A} 3$ and $\mathrm{A} 4$.

The comparison of data and parameters directly shows that $\mathrm{Mg}^{2+}$ ions cause a significant global conformational change of the molecule to a more compact conformation (the radius of gyration $R_{\mathrm{g}}$ decreases by $\sim 15 \%$ and the maximum diameter $D_{\max }$ by $\sim 25 \%$ ). This is also true for the complexes of tmRNA with SmpB. Similar conformational changes are observed upon SmpB addition in the absence of $\mathrm{Mg}^{2+}$ ions, though of reduced amplitude, with $\sim 7 \%$ reduction in $R_{\mathrm{g}}$ and almost $20 \%$ in $D_{\text {max }}$. As expected for solutions eluting from a SE column, molecular mass estimates derived from the intensity at the origin are close to the values calculated from tmRNA and SmpB sequences. This is further confirmed by the values of the hydrated molecular volume derived from the Porod expression, all of which are compatible with a monomer of tmRNA (respectively, complex with SmpB).

\section{Rigid-body modeling}

TmRNA is a highly structured molecule comprising eight modules. We, therefore, decided to use a rigid-body modeling approach as implemented in the SASREF program (Petoukhov and Svergun 2005) using the components of the pseudo-atomic model proposed by Ramrath et al. (2012) as defined in Table 2 and explained in detail in the molecular modeling section of Materials and Methods. In order to avoid any bias in the computation from their starting spatial localization, all eight modules were superimposed at the origin.

For each sample, 300 models were obtained that fitted the experimental data within experimental uncertainties. The
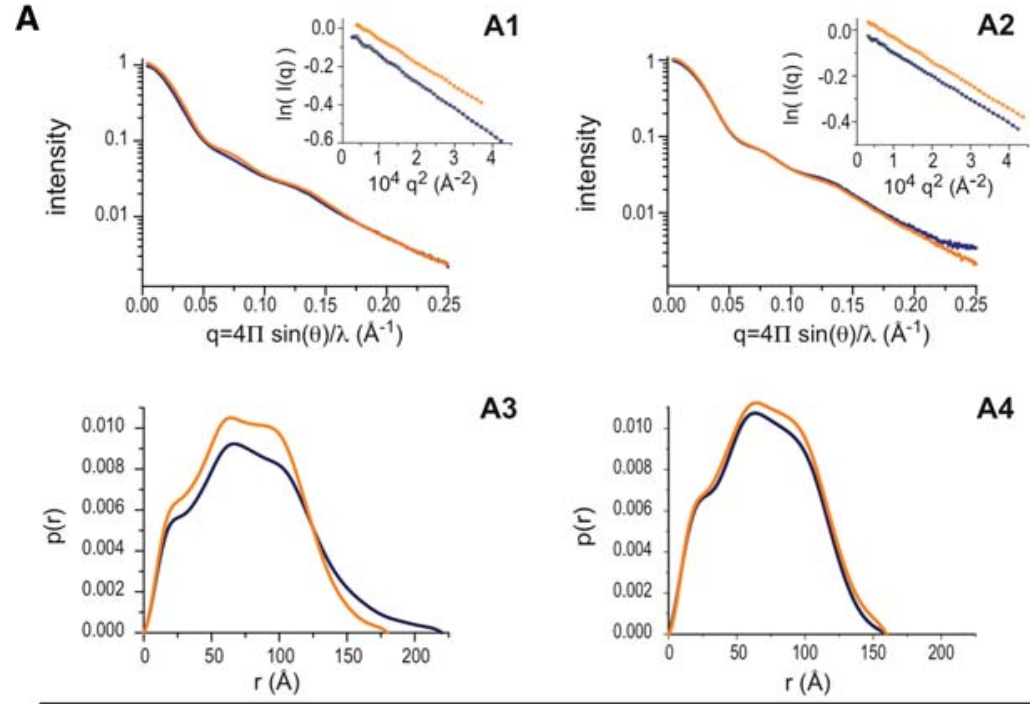

A3

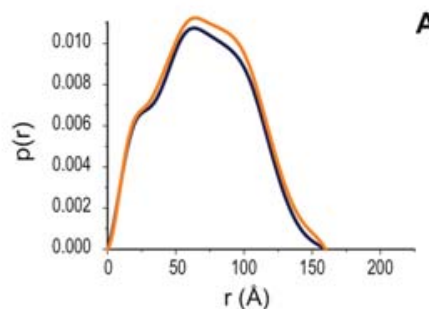

A4

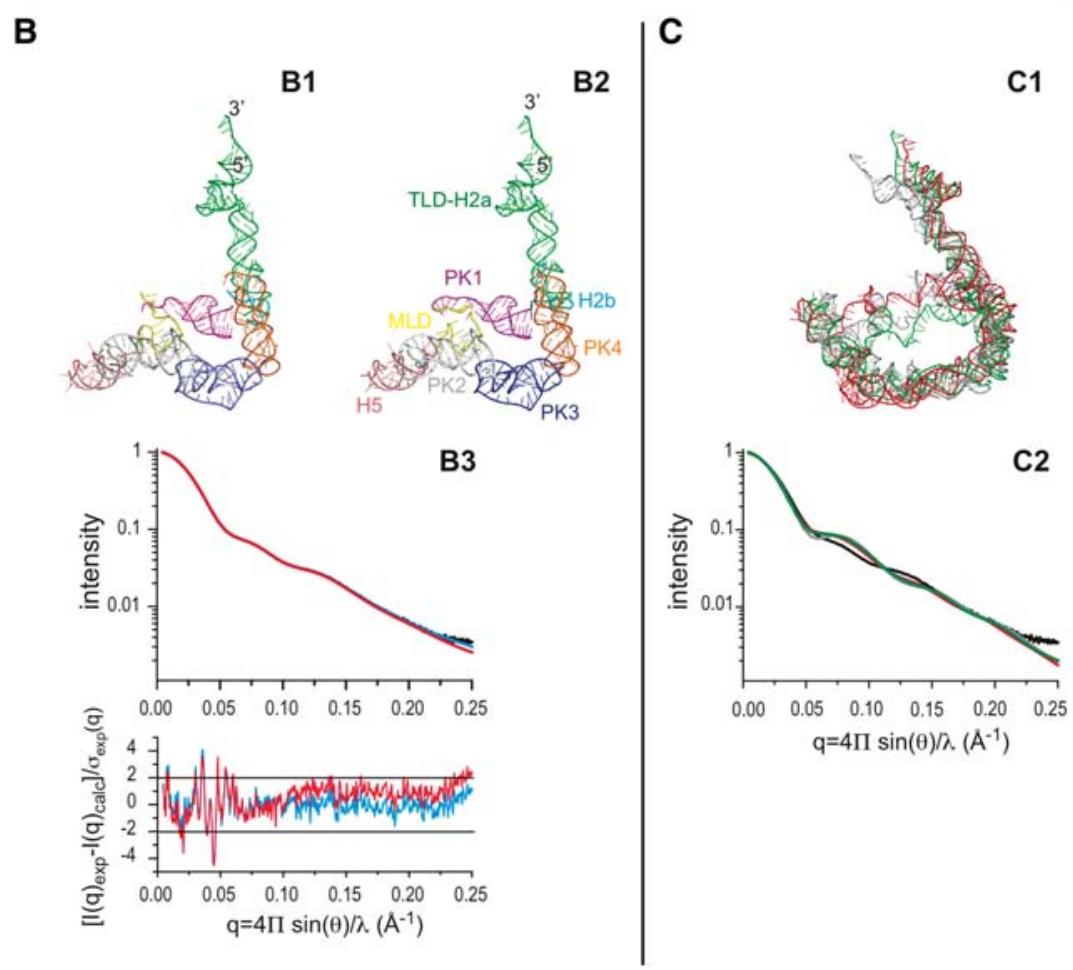

FIGURE 6. SAXS study of tmRNA and tmRNA:SmpB complex in solution. (A) SAXS data analysis. (Blue line) tmRNA alone, (orange line) tmRNA-SmpB complex (1:1 stoichiometry). (A1) SAXS patterns recorded in the absence of $\mathrm{Mg}^{2+}$ ions; (A2) SAXS patterns recorded in the presence of $5 \mathrm{mM} \mathrm{Mg}^{2+}$; (insets) Corresponding Guinier plots; (A3) $p(r)$ functions derived from intensities recorded in the absence of $\mathrm{Mg}^{2+} ;(A 4) p(r)$ functions derived from intensities recorded in the presence of $5 \mathrm{mM} \mathrm{Mg}^{2+}$. (B) 3D models obtained using SASREF in the case of tmRNA alone in the presence of $\mathrm{Mg}^{2+}$. (B1) Model from SASREF; (B2) the same model after connectivity restoration using ModeRNA; (B3) scattering patterns: experimental data (black), B1 SASREF models (cyan), B2 model after connectivity restoration (red). The corresponding reduced residuals are shown in the bottom graph (same color code as above). (C) Cryo-electron microscopy-derived models do not fit the experimental data. (C1) The three models have been superimposed over the loop residues. (Red) 3J18, (green) 3iyq, (gray) $20 b 7$ (see text for details); (C2) calculated scattering patterns of the three models superimposed over the experimental data in the presence of $\mathrm{Mg}^{2+}$, in black. Same color code as in $\mathrm{C1}$. 
TABLE 1. Parameter values obtained from the analysis of SAXS scattering curves

\begin{tabular}{llccccc}
\hline & & $R_{\mathrm{g}}$ Guinier $(\AA)$ & MW seq $(\mathrm{kDa})$ & $\mathrm{MW} /(0) / \mathrm{c}(\mathrm{kDa})$ & $R_{\mathrm{g}} p(r)(\AA)$ & $D_{\max }(\AA)$ \\
\hline $\mathrm{No} \mathrm{Mg}^{2+}$ & tmRNA & $64.8 \pm 0.5$ & 117 & $120 \pm 10$ & $63.5 \pm 0.5$ & 220 \\
& $1-1$ complex & $60.8 \pm 0.5$ & 132 & $138 \pm 10$ & $58.9 \pm 0.5$ & 180 \\
$5 \mathrm{mM} \mathrm{Mg}^{2+}$ & tmRNA & $55.4 \pm 0.5$ & 117 & $130 \pm 10$ & $54.6 \pm 0.5$ & 160 \\
& $1-1$ complex & $55.5 \pm 0.5$ & 132 & $149 \pm 10$ & $55.8 \pm 0.5$ & 160 \\
\hline
\end{tabular}

most striking observation is the unique arrangement of modules, with the pseudoknots, the MLD and H5 arranged in a large loop, while $\mathrm{H} 2$ acts like a hinge with the TLD pointing away from the loop as shown in Figure 6B, panel B1 that presents the direct result of one rigid body refinement in the case of tmRNA alone in the presence of $\mathrm{Mg}^{2+}$ ions. The same model is displayed in panel B2 after restoration of backbone connectivity, while the corresponding scattering patterns are superimposed over experimental data in panel B3. Reduced residual plots are also presented and attest to the quality of the model. This is very reminiscent of the model extracted from the ribosome-tmRNA-SmpB complex and is remarkable in view of the starting point for refinement in which all domains are superimposed at the origin. Under all conditions, models are found in which the H2-TLD "arm" is found on either side of the loop, and models with the correct orientation were selected before further analysis (see Materials and Methods for details).

\section{Statistical analysis of tmRNA structural parameters}

From a mere visual inspection of models, the loop domains do not exhibit major rearrangements in the presence of $\mathrm{Mg}^{2+}$ that could explain the more compact overall shape suggested by the structural parameters $R_{\mathrm{g}}$ and $D_{\max }$ (Table 1). To try and detect some conformational differences that could be correlated with the observed differences in $R_{\mathrm{g}}$ and $D_{\max }$ values, we undertook a few systematic calculations on all models. To characterize the shape and size of the loop, we calculated the distribution of distances between the center of mass of the loop and all atoms of the loop domains. All distributions corresponding to the same sample were then averaged, and the averages are plotted in Supplemental Figure 4A, panel A1. While the curves derived from models in the absence of $\mathrm{Mg}^{2+}$ are asymmetric, corresponding to an anisometric shape of the loop, those in the presence of $\mathrm{Mg}^{2+}$ appear to be distinctly more symmetric. Furthermore, the root mean square distance undergoes a clear reduction from $50.4 \AA$ to $46.1 \AA$ in the presence of $\mathrm{Mg}^{2+}$. In the absence of $\mathrm{Mg}^{2+}$, $\mathrm{SmpB}$ addition shifts the distribution toward smaller values by $\sim 1 \AA$ without changing its shape. This explains, at least in part, the observed reduction in the radius of gyration of the molecule upon SmpB and $\mathrm{Mg}^{2+}$ addition. We also calculated the distribution of the distances of all atoms of the loop to the "mid-plane" (defined by the first two inertia axes of the loop) averaged over all models for a given condition (Supplemental Fig. 4A, panel A2). In the presence of $\mathrm{Mg}^{2+}$ ions, distributions are narrower around zero than in the absence of $\mathrm{Mg}^{2+}$, showing that the loop exhibits excursions of reduced amplitude normal to the mid-plane. In conclusion, the loop appears to be more plane and isometric in the presence of $\mathrm{Mg}^{2+}$.

We then turned to investigate the orientation of the TLD with respect to the loop using two parameters determined with respect to the mid-plane of the loop. First, $\alpha$ is the angle between the vector linking the center of mass of the loop $\mathrm{O}$ and the projection on the mid-plane $\mathrm{M}$ of the hinge point of the H2 domain with the loop (phosphorus atom 304) and the vector MP where $\mathrm{P}$ is the projection on the mid-plane of phosphorus atom 363 at the far end of the TLD (see diagram in Supplemental Fig. 4A, panel A3). Second, $Z$ is the $Z$-coordinate of phosphorus 363 that reports about the elevation of the TLD. The mean values of each parameter with associated standard deviations are plotted in Supplemental

TABLE 2. Structural rigid modules used in tmRNA 3D structure reconstruction in SASREF

\begin{tabular}{|c|c|c|c|c|c|c|c|c|}
\hline \multicolumn{2}{|c|}{ Eight rigid modules } & \multirow{2}{*}{$\begin{array}{l}\text { Boundaries } \\
1-42311-363\end{array}$} & \multicolumn{2}{|c|}{ Three rigid modules } & \multirow{2}{*}{$\begin{array}{l}\text { Boundaries } \\
1-42 \text { 311-363 }\end{array}$} & \multicolumn{2}{|c|}{ Four rigid modules } & \multirow{2}{*}{$\begin{array}{l}\text { Boundaries } \\
1-48301-363\end{array}$} \\
\hline 1 & $\mathrm{TLD}+\mathrm{H} 2 \mathrm{a}(+S m p B)^{\mathrm{a}}$ & & 1 & $\mathrm{TLD}+\mathrm{H} 2 \mathrm{a}(+S m p B)^{\mathrm{a}}$ & & 1 & $\mathrm{TLD}+\mathrm{H} 2 \mathrm{a} \mathrm{b}(+S m p B)^{\mathrm{a}}$ & \\
\hline 2 & $\mathrm{H} 2 \mathrm{~b}$ & $43-48$ 301-310 & 2 & $\mathrm{H} 2 \mathrm{~b}$ & $43-48$ 301-310 & 2 & PK1 & $49-83$ \\
\hline 3 & PK1 & $49-83$ & 3 & PK1 & $49-300$ & 3 & MLD & 84-104 \\
\hline 4 & MLD & 84-104 & & MLD & & 4 & $\mathrm{H} 5$ & $105-300$ \\
\hline 5 & $\mathrm{H} 5$ & 105-137 & & H5 & & & PK2 & \\
\hline 6 & PK2 & 138-198 & & PK2 & & & PK3 & \\
\hline 7 & PK3 & 199-246 & & PK3 & & & PK4 & \\
\hline 8 & PK4 & $247-300$ & & PK4 & & & & \\
\hline
\end{tabular}

${ }^{\mathrm{a}} \mathrm{SmpB}$ from 1P6V has been positioned in interaction with the TLD as observed in the crystal structure (PDB: 1P6V) (Gutmann et al. 2003). 
Figure 4, panels A4 and A5. a exhibits a marked decrease from $\sim 110^{\circ}$ down to $\sim 60^{\circ}$ upon $\mathrm{Mg}^{2+}$ addition, while, in contrast, the distance of the end point of TLD to the mid-plane increases from an average value of $80 \AA$ to an average distance of 100 $\AA$. Therefore, the TLD appears to be positioned more directly above the center of the loop (smaller $\alpha$ ) and with its extremity higher above the mid-plane (higher $Z$-value).

The existence of correlations between $\alpha$ and $Z$ values was investigated in a coarse-grained manner using the heat maps shown in Supplemental Figure 4B. In the absence of $\mathrm{Mg}^{2+}, Z$ and a exhibit essentially monomodal distributions peaking around $70 \AA$ and $130^{\circ}$, respectively, with more than half of all models comprised within the gray box [40 $\AA$, $100 \AA]_{Z}$ and $\left[100^{\circ}-160^{\circ}\right]_{\alpha}$ (Supplemental Fig. $4 \mathrm{~B}$, upper panel). In contrast, in the presence of $\mathrm{Mg}^{2+}$, a rectangular cornershaped distribution is observed around a square center [100 $\AA, 140 \AA]_{Z}$ and $\left[40^{\circ}-80^{\circ}\right]_{\alpha}$ with a horizontal extension [120 $\AA, 140 \AA]_{Z}$ extending down to $0^{\circ}$ for $a$ and a vertical one $\left[60^{\circ}-80^{\circ}\right]_{\alpha}$ down to $40 \AA$ for $Z$ (Supplemental Fig. $4 \mathrm{~B}$, lower panel). The gray box contains two thirds of all models.

Clustering offers another way to organize the ensemble of solutions; they were grouped in similarity clusters based on RMSD calculations using the routine Damclust (Petoukhov et al. 2012). In the absence of $\mathrm{Mg}^{2+}, 10$ clusters are identified, five of which account for $75 \%$ of all solutions (the location of each centroid is represented as a star in Supplemental Fig. 4B, upper panel). In the presence of $\mathrm{Mg}^{2+}$, four main clusters out of nine account for $78 \%$ of all models (Supplemental Fig. 4B, lower panel). The distributions of centroids show a good overlap with both heat maps.

The conclusion of this coarse-grained analysis of our models is that the addition of $\mathrm{Mg}^{2+}$ to the solution of tmRNA causes a small but clear compaction of the loop, which becomes flatter and more isometric together with a movement of the TLD centered above the loop and mainly away from the mid-plane of the loop.

\section{Comparison with cryo-EM-derived models}

In a complementary approach to the previous bias-free, rigid-body analysis, we tried to evaluate existing models derived from cryo-EM studies of tmRNA and SmpB complexed with ribosomes and other factors representing different steps of the tmRNA rescue task: pre-accommodation (Kaur et al. 2006), accommodation (Weis et al. 2010), and post-translocation (Fig. 6C, panel C1; Ramrath et al. 2012). Scattering patterns were calculated and are shown in Figure 6C, panel C2. No model provides even a decent fit to our experimental data on tmRNA in $5 \mathrm{mM} \mathrm{Mg}^{2+}$ containing buffer (the cryoEM samples contained $\mathrm{Mg}^{2+}$ ions) with $\chi$ values comprised between 17.5 and 21 . Thus, although the topology and overall shape of tmRNA is basically unchanged between the solution state and the complex with ribosome, tmRNA undergoes large amplitude conformational changes upon ribosome binding and through the various stages of its task.
Cryo-EM data suggest that major tmRNA conformational rearrangements during trans-translation steps on the ribosome involve changes in the relative orientation of the TLD$\mathrm{H} 2$ with respect to the big loop. A second trial was thus conducted with only three rigid modules, the TLD, H2, and the loop, which was "frozen" in the conformation of the posttranslocation step (PDB 3J18; 12). The fits between the computed and the experimental data recorded with $\mathrm{Mg}^{2+}$ were improved compared to the initial models but fell short of an acceptable fit, with $\chi$ values on the order of 5 .

According to NMR, the MLD solution structure composed of a small stem-loop framed by two single strands is much less constrained that those of the pseudoknots. In a third step, we, therefore, released the MLD module from both PK1 and H5 and used four rigid modules (TLD-H2, PK1, MLD, H5PK4). The fit between the model calculated curves and the experimental data recorded in $\mathrm{Mg}^{2+}$ keeps improving with the increase in the number of degrees of freedom ( $\chi$ values between 1.6 and 2). Interestingly, the $(\alpha, Z)$ distribution analysis shows that most solutions are grouped into the low $a-$ low $Z$ quarter of the map, in marked contrast to that observed on the models obtained starting with all modules at the origin (Supplemental Fig. 4). Keeping all four PKs in the position found in the model proposed by Ramrath et al. (2012) constrains the H2-TLD module to adopt a different set of positions as characterized by the $(\alpha, Z)$ distribution that, besides, lead to fits of lower quality. This suggests that the PKs are arranged differently in solution, which is not unexpected as the conformation of the loop in the cryo-EM model results from its numerous interactions with various parts of the bound ribosome, some of which are actually located inside the loop.

\section{DISCUSSION}

\section{Origin of the systematic aa-tmRNA degradation in $E$. coli cells}

How and why does aa-tmRNA, composed of very stable structural blocks that should resist to temperatures up to $95^{\circ} \mathrm{C}$, undergo systematic and reproducible degradation in E. coli? The precise identification of the set of RNases responsible for our patterns of degradation would require testing the overproduction of aa-tmRNA in several E. coli strains deleted for several RNase genes. This ongoing work is beyond the scope of this study.

In the first place, we can imagine that degradation leading to aa-tmRNA-D2 can arise from multiple pathways, including degradation from ill-folded conformers, degradation from aa-tmRNA-D1, or degradation from abortive transcripts followed by digestion of the sequence upstream of $\mathrm{H} 5$ and downstream from PK4. Coproduction assays demonstrate that SmpB prevents formation of aa-tmRNA-D2, so the last hypothesis is valid if SmpB protects the neosynthetized single strand during transcription. For reasons that are exposed 
hereafter, we suspect that aa-tmRNA-D2 mostly originates from full transcripts ill-folded in the TLD region, which would destabilize and expose parts of the RNA that are naturally protected from the action of RNAses in well-folded molecules. Despite its heterologous origin, the A. aeolicus TLD (aa-TLD) can be overproduced in high quantity using the same plasmid and E. coli strain (Gaudin et al. 2003), showing that it is mostly well-folded and not degraded. In contrast, we observed that no production of a longer aa-tmRNA derivative mimicking both the aa-TLD and the connecting stem $\mathrm{H} 2$ could be obtained. The $\mathrm{H} 2$ sequence itself and the proper association of its two strands would thus be critical for proper TLD folding and vice versa. The GC content of the aa-ssrA gene is higher than that of E. coli, in particular in the TLD arms and in helix $\mathrm{H} 2$. It has evolved to favor active $3 \mathrm{D}$ folds at high temperature. It is thus not surprising that, at $37^{\circ} \mathrm{C}$, a number of competing alternate structures can be produced in high amounts and be readily degraded by the host RNases. Such structures are not recognized as tRNAs and may readily undergo degradation by the host RNases. Degradation of aatmRNA conformers, either full-length or $\triangle \mathrm{MLD}$, with misfolded TLD, can thus explain band D2. This observation is also reminiscent of the degradation of the unprotected $3^{\prime}$ end of the coding strand in Caulobacter crescentus two-pieces tmRNA by RNase R (Hong et al. 2005). Such band is not observed in the case of $E$. coli either because there is no ill-folded ec-tmRNA molecule in E. coli, as suggested by Hong et al., or because ill-folded conformers exist, but $E$. coli pseudoknots are not strong enough to resist degradation by endogenous RNases. Recently, Lian and Deutscher (2012) showed that SmpB regulates the RNase $\mathrm{R}$ turnover and that the efficiency of the regulation is enhanced when $\mathrm{SmpB}$ is complexed to tmRNA. We are currently investigating the role of RNase $\mathrm{R}$ in aa-tmRNA degradation.

The origin of band D1 was, at first glance, more intriguing. PAGE and MS analyses provide evidence for a degradation product resulting from endonucleolytic cleavages and lacking the MLD region. The presence of the $3^{\prime}$-end region of aatmRNA is revealed by the methyl resonance on the NOESY spectrum. In E. coli, most RNAs are digested in a stepwise fashion, starting with an internal endonucleolytic cleavage followed by exonucleolytic digestion. The observation of aatmRNA-D1 is consistent with such a degradation pathway. RNase levels in the cell can be up-regulated as a result of the cellular stress caused by overproduction of a heterologous tmRNA. The endotoxin MazF that targets single-stranded ACA triplets (Zhang et al. 2003) is a potential candidate for the initial endonucleolytic cut of the MLD region. It was shown to degrade successfully mutant ec-tmRNA bearing ACA triplets (Baik et al. 2009). Our gels show that a slight proportion of full-length tmRNA is recovered, probably because, in the cell, oligomeric association protects the MLD from degradation. As a conclusion, the unexpected observation of tmRNA digests D1 and D2 was made possible because of the exceptional stability of the blocks composing the aa-
tmRNA. We suspect that similar degradation pathways might exist for every heterologous tmRNA and also probably for the ec-tmRNA and that these pathways are important for the quality control of tmRNA structure in vivo and maybe for regulation of the number of functional tmRNA molecules in the cell. Heterologous production of tmRNA in E. coli thus provides a new tool to study these degradation pathways.

\section{SmpB binding site of highest affinity from in vivo protection}

The observation and characterization of aa-tmRNA-D1 and aa-tmRNA-D2 show that RNases stop when encountering strong structural blocks. These digests can be used as original tools to probe in vivo the localization of the first SmpB binding event onto tmRNA. Endogenous SmpB and tmRNA are produced in vivo in similar amounts (Sundermeier and Karzai 2007). This suggests that in vivo, outside the ribosome, most free tmRNA molecules will associate with a single SmpB. UV shadowing is quantitative and discloses a slight increase of the relative proportion of full-length aa-tmRNA with respect to digest D1 (Fig. 2). This suggests that SmpB converts illfolded aa-tmRNAs into good tRNA-like structures. SmpB binding does not protect aa-tmRNA from the endonucleolytic degradation leading to D1 digest as the His6-MS2 coat protein dimer does (Fig. 5). SmpB is slightly larger than one monomer of the His6-MS2 coat protein dimer (157 aa vs. $2 \times 138$ aa). Steric hindrance resulting from SmpB binding in the PK1-MLD region would thus be expected to prevent MLD from endonuclease attacks. This is not the case. The RNA extract pattern of the coproduction assay with $\mathrm{SmpB}$ is thus fully understood if we suppose that TLD binds as observed in the crystallographic structures (Gutmann et al. 2003; Bessho et al. 2007; Ramrath et al. 2012). The absence of degradation from alternative wrong folds confirms the assumption that SmpB exerts a chaperoning action on TLD folding and provides a structural explanation to the observed enhanced aminoacylation in the presence of SmpB.

Former probing experiments established the existence of at least two SmpB binding sites, one on the TLD and the other one within the necklace formed by the PKs and the MLD (Wower et al. 2002; Konno et al. 2007). The respective dissociation constants measured on tmRNA fragments recapitulating the two binding sites are $\sim 40 \mathrm{nM}$ and $2 \mathrm{nM}$ (Metzinger et al. 2008). This apparent inconsistency with our in vivo protection results and with a recent study reporting a $K_{\mathrm{d}}$ of 0.4 $\mathrm{nM}$ in $100 \mathrm{mM} \mathrm{Na}^{+}$and $2.6 \mathrm{nM}$ in $10 \mathrm{mM} \mathrm{Mg}^{2+}$ on a synthetic two-stranded TLD (Fahrat and Rueda 2012) could arise from the nature of the RNA samples under investigation. The former full-length aa-tmRNA was transcribed in vitro, a method conveniently avoiding RNases attacks, and purified in denaturing conditions. The resulting tmRNA thus lacks the two post-transcriptional modifications ( $\mathrm{T}$ and $\Psi$ ) that stabilize the TLD structure. The affinity of SmpB for the TLD of fulllength tmRNA in vivo is probably better than in the in vitro 
context. The second drawback of the in vitro RNA production is intrinsically linked to the length of the RNA. The neosynthesized RNA folds as transcription proceeds. In vitro, the aforementioned quality control of the structure does not exist, and ill-folded tmRNAs are not degraded. The in vitro tmRNA sample is, thus, certainly much more conformationally heterogeneous than its in vivo counterpart purified in native conditions. Our gel filtrations indicated the existence of a mixture of aggregates, dimers, and a slight proportion of monomers, even after thermal treatment similar to that used by Metzinger et al. (2008). The $K_{\mathrm{d}}$ values reported were most probably measured on a heterogeneous population of tmRNA conformers and oligomers. Konno et al. (2007) have shown that the C-ter tail-truncated SmpB protects U85 of ec-tmRNA from chemical modification, i.e., in a region consistent with the binding site of highest affinity observed in vitro (Metzinger et al. 2008). However, the ec-tmRNA sample was subjected to isopropylic precipitation, and in such conditions, we were not able to get monodispersed sample. According to our experimental models, the SmpB molecule bound to the TLD is too far away from U85 to interact directly. If this molecule effectively conferred protection, then it must be indirect. However, we cannot rule out that a small proportion of SmpB binds in vivo to the tmRNA loop, which would be hidden by the major fraction binding TLD.

It has been proposed that $\mathrm{SmpB}$ and protein $\mathrm{S} 1$ would act in concert as a scaffold for tmRNA (Gillet et al. 2007; Felden and Gillet 2011). A preferential binding of SmpB on TLD leaves open the access of the tmRNA loop for S1 binding (Wower et al. 2000). Also, one SmpB molecule can bind the empty decoding site of free $70 \mathrm{~S}$ ribosome in the absence of tmRNA in vivo (Gillet et al. 2007). The authors proposed that, depending on environmental conditions, several pathways of tmRNA recruitment are likely to initiate trans-translation (Felden and Gillet 2011). The most recent cryo-EM structure of the preaccommodation state shows a TLD complexed to two SmpB molecules binding the GTPase-associated center of the $50 \mathrm{~S}$ and the decoding center of the $30 S$ (Kaur et al. 2006), respectively. Our finding that SmpB binds preferentially the TLD in vivo is consistent both with the most recent crystal structure of the pre-accommodation state which shows the TLD complexed to a single SmpB molecule that also binds the decoding center of the 30S (Neubauer et al. 2012) and with the existence of an alternative recruitment pathway of tmRNA by a 70S ribosome preloaded with one $\mathrm{SmpB}$ in the decoding site, as first suggested by Hallier et al. (2004).

\section{Structural insights into the [tmRNA.SmpB] complex}

Despite the existence of alternative recruitment pathways (Felden and Gillet 2011), several data suggest that tmRNA is most likely recruited by ribosome as a complex. Our goal was to shed some light on the conformation of both tmRNA and [tmRNA.SmpB] (1:1) complex in solution before ribosome binding. It is not possible to discriminate be- tween the two SmpB binding sites on the basis of the SAXS data alone because of the too small contribution of the protein to the total scattered intensity. For our SAXS modeling, SmpB was positioned in interaction with the TLD as found in the crystal structure of the complex (Bessho et al. 2007) and in accordance with our in vivo probing. The structural parameters measured on [tmRNA.SmpB] complexes by SECSAXS show that a significant compaction of the molecule occurs upon SmpB binding (Table 1). We computed rigid-body models of tmRNA and [tmRNA.SmpB] complex using the eight modules (Table 2) used by Ramrath et al. (2012) in the presence or in the absence of $\mathrm{Mg}^{2+}$. All resulting models that yield an excellent match with the experimental data exhibit the loop made of the pseudoknots and the MLD, connected to the TLD-H2 region which points outward. The presence of $\mathrm{Mg}^{2+}$ ions is associated with a more circular and flatter loop conformation together with a TLD arm rising higher above the loop.

In contrast, the scattering curves computed from the cryoEM structures of the tmRNA at various steps of trans-translation display major discrepancies with the experimental data (Fig. 6C, panel C2). This shows that, although the topology appears to be preserved, the tmRNA conformation before ribosome binding is different from that in all studied ribosomebound states. In particular, our models suggest that the large loop adopts a more compact conformation in the free state than in complex with the ribosome. Although the tightly folded conformation proposed by Ivanov et al. (2002) is not supported by our data, the basic idea of a more compact conformation in isolation, also suggested by Burks et al. (2005), holds true but in a more subtle way, the various conformations explored by the tmRNA in solution and later in the different stages of its activity appearing more like variations on a common topological theme.

\section{CONCLUSION}

Our results provide experimental information on the conformation of [tmRNA.SmpB] complex in solution before ribosome binding. TmRNA appears to be self-structured, even as a free molecule, with a characteristic topology that makes it mechanically easy to bind ribosome and that can be modulated through its interactions with various parts of the ribosome and associated factors. We showed by original in vivo protection assays that the first SmpB preferentially binds to the TLD and that the binding causes a significant compaction of the entire tmRNA in the absence of $\mathrm{Mg}^{2+}$ ions. The study of the degradation pattern of heterologous RNAs that evolved to withstand high temperature thus constitutes an original and valuable tool to monitor the relative stability of the different parts of the molecules in vivo and to get the footprints of protein partners in vivo.

Our overproduction assays show that the self-structuration of tmRNA explains its high resistance to RNases and thereby its exceptional longevity in a functional state within the cell. 
By overproducing the heterologous aa-tmRNA in E. coli, we have, in a way, mimicked drastic modifications of the selection pressure. The degradation of the heterologous aatmRNA also demonstrates that tmRNA sequence was finely tuned by evolution to ensure its stability, even in the absence of any protein partner. However, this does not mean that SmpB has no stabilizing or chaperoning effect on tmRNA structure. Indeed, it was formerly observed that the overproduction of tmRNA with its protein partner SmpB allows the recovery of higher amounts of full-length aa-tmRNA (Hallier et al. 2004). SmpB is known to enhance TLD aminoacylation (Barends et al. 2001; Shimizu and Ueda 2002). HanawaSuetsugu et al. (2002) suggested that it proceeds by modulating TLD conformation to enhance recognition by AlaRS. The authors also showed that the protein protects nonaminoacylable mutant TLD from degradation in vivo. The disappearance of the aa-tmRNA-D2 species resulting from our co-overproduction assays brings an original piece of evidence that $\mathrm{SmpB}$ chaperones the TLD structure.

\section{MATERIALS AND METHODS}

\section{Expression tests and RNA recovery}

Either an XL1 or JM101tr E. coli strain was used in all expression tests. Expression tests for total RNA extracts recovery were performed in $20 \mathrm{~mL} \mathrm{LB}$ medium. Cells were pelleted and resuspended in $400 \mu \mathrm{L}$ of $10 \mathrm{mM}$ magnesium acetate, $10 \mathrm{mM}$ Tris- $\mathrm{HCl}, \mathrm{pH}$ 7.4. Protein-free total RNA extracts were recovered after phenol/chloroform extraction and ethanol precipitation as described before (Ponchon et al. 2009). They were analyzed by SDS-PAGE electrophoresis. Bands were visualized by UV shadowing.

\section{RNA production and purification}

Cells transformed with pBSTNAV, pProRNA, or p44K plasmids were grown in either LB or 2XTY growth medium and harvested as described before (Ponchon et al. 2013). The RNAs were recovered following protocols described by Ponchon et al. (2009). The aqueous phase was purified in four steps by FPLC using nondenaturing buffers. TmRNA samples were first separated from the bulk of tRNAs and 5S RNA on a source 15Q (GE Healthcare) pre-equilibrated in $\mathrm{H}_{2} \mathrm{O}$, potassium phosphate $\mathrm{pH} 6.5,20 \mathrm{mM}$, and EDTA $1 \mathrm{mM}$ using a $50 \mathrm{mM}-1 \mathrm{M} \mathrm{NaCl}$ elution gradient. Purity was further refined on a more resolutive mono $\mathrm{Q}$ column using the same loading and elution buffers and a $200-500 \mathrm{mM} \mathrm{NaCl}$ gradient. Last contaminants were eliminated using a hydrophobic column. A final round of gel filtration (Superdex 200, Amersham) was used to remove aggregates prior to NMR and SAXS experiments. Analytical gel filtrations were performed at $17^{\circ} \mathrm{C}$ using an analytical Superdex 200 column and $150 \mathrm{mM} \mathrm{NaCl}, 20 \mathrm{mM}$ Tris $\mathrm{HCl}$, pH 6.5 elution buffer with ( $1 \mathrm{mM}$ ) or without $\mathrm{MgCl}_{2}$ added. RNAs extracts and purification fractions were analyzed by either SDS or $8 \mathrm{M}$-urea PAGE ( $10 \%$ or $12 \%)$. RNA bands were visualized by UV shadowing (Ponchon et al. 2009). SDS-PAGE provides good resolution in the range of our RNAs of interest and is time saving ( $1 \mathrm{~h}$ migration at $200 \mathrm{~V}$, TGS loading buffer). To improve resolution over the full range of interest (60-400 nt), we sometimes used $4 \%-12 \%$ gradient Bis Tris gels (NuPAGE Novex) with NuPAGE MES SDS running buffer and NuPAGE LDS sample buffer.

\section{Hybridization experiments}

Aa-tmRNA fragments were heated for $2 \mathrm{~min}$ at $98^{\circ} \mathrm{C}$ in water with $2 \mu \mathrm{L}$ of a $100-\mu \mathrm{M}$ DNA oligonucleotides anti-H2PK1 (15 nt, $5^{\prime}$ ACCCGGCCTGCTCCT) or anti-TLD (35 nt, 5'-AAATAACTTTC TGCAGTGGTGGAGGCGGCGGGAAT) water solution for final volumes of $15 \mu \mathrm{L}$ and cooled down to $4^{\circ} \mathrm{C}$ in $30 \mathrm{sec}$. Only the last $19 \mathrm{nt}$ of the anti-TLD are complementary to nucleotides 329 to 347 of aa-tmRNA. Band shifts were assayed on 12\% SDS PAGE. Samples were loaded on the gels at room temperature.

\section{NMR}

The purified aa-tmRNA-D1 was deacylated in Tris buffer, $\mathrm{pH} 8$ at $37^{\circ} \mathrm{C}$ for $1 \mathrm{~h}$ and then dialyzed several times against $1 \mathrm{mM}$ EDTA water solutions and finally extensively against water. Synthetic aaMLD and ec-MLD oligonucleotides were purchased from Dharmacon Inc. The unprotection buffer was exchanged against water on G25 desalting column (GE Healthcare). All samples were freezedried before resuspension in $95 \% \mathrm{H}_{2} \mathrm{O}-5 \% \mathrm{D}_{2} \mathrm{O}$ and $0.01 \mathrm{mM}$ EDTA. The $\mathrm{pH}$ was adjusted to 6.5 by addition of small aliquots of $0.1 \mathrm{~N} \mathrm{NaOH}$ under stirring. The purified aa-tmRNA-D1 strand concentration was $0.1 \mathrm{mM}$. Synthetic oligonucleotide concentrations range from 0.2 to $1 \mathrm{mM}$. All NMR spectra were acquired in $5 \mathrm{~mm}$ Shigemi tubes at $600 \mathrm{MHz}$ on a Bruker Avance DRX600 spectrometer equipped with a cryoprobe and $Z$-axis gradient. Water signal in 1D and 2D NOESY experiments was either nonexcited using the Jump and Return (jr) procedure (Guéron et al. 1991) or suppressed using the W5 Watergate (wg) pulse sequence (Liu et al. 1998). The resonances were assigned using the usual set of $2 \mathrm{D}$ homonuclear NMR methods in $\mathrm{D}_{2} \mathrm{O}$ and in $95 \% \mathrm{H}_{2} \mathrm{O} / 5 \% \mathrm{D}_{2} \mathrm{O}: 2 \mathrm{D}$ NOESY with 60-, 90-, 150-, and 300-msec mixing times, 2D TOCSY, 2D ${ }^{1} \mathrm{H}^{15} \mathrm{~N}$-jr-HSQC.

\section{MS experiments}

RNA and protein sample buffers were exchanged against $150 \mathrm{mM}$ ammonium acetate buffer, $\mathrm{pH} 6.5$ using microcentrifuge gel-filtration columns (Zeba $0.5 \mathrm{~mL}$, Thermo Scientific). Concentrations were determined spectrophotometrically using absorbance at 260 and $280 \mathrm{~nm}$, respectively. RNA mass measurements under denaturing conditions were performed on a quadrupole/time-of-flight instrument ( $\mu \mathrm{Q}$-TOF, Bruker) operating in the negative ion mode. Samples were diluted down to $2 \mu \mathrm{M}$ in H2O/ACN/TEA (50/50/1). Experiments under nondenaturing conditions were performed on a time-of-flight instrument (LCT, Waters) equipped with an automated chip-based nanoESI source (Triversa Nanomate, Advion Biosciences) operating in the positive ion mode. Data analysis was performed with MassLynx 3.5 (Waters).

\section{Sample preparation for SAXS}

SmpB is extremely well conserved. It is able to successfully complement heterologous strains deleted for their endogenous protein 
(Thibonnier et al. 2010). Therefore, ec-tmRNA and aa-SmpB can be used together for structural investigations. $\Delta$ mutants in the C-ter tail are functionally inactive but retain high affinity for tmRNA (Jacob et al. 2005; Sundermeier et al. 2005; Hallier et al. 2006) and are much easier to purify. The protein was purified by FPLC on phenyl sepharose and a monobed anion exchange Resource $S$ column (GE Healthcare). After concentration on Amicon Ultra-15 3K (Millipore), the buffer was exchanged for the SAXS buffer and the purification of the protein sample polished on a Superdex 75 column (GE Healthcare) preequilibrated with the SAXS buffer. In order to control the stoichiometry of each partner, ec-tmRNA and aa- $\Delta 16$ $\mathrm{SmpB}$ (without the $16 \mathrm{C}$-ter amino acids) were separately produced in E. coli jM101tr and RosettaTM (DE3)pRARE2 strains, respectively, and purified in native conditions before mixing. The literature reports $K_{\mathrm{d}}$ values on the order of $1 \mathrm{nM}$ in the presence of 5-10 mM $\mathrm{Mg}^{2+}$, derived from SPR (Hallier et al. 2006), fluorescence anisotropy (Fahrat and Rueda 2012), filter-binding assays (Jacob et al. 2005), and from gel mobility-shift assays (Sundermeier et al. 2005). The protein and RNA concentrations used for this experiment are on the order of $2-25 \mu \mathrm{M}$, and consequently the proportion of free protein in the mixture is negligible.

\section{SAXS experiments}

$\mathrm{X}$-ray scattering data were recorded at Synchrotron SOLEIL (Gifsur-Yvette, France) on the SWING beamline. The wavelength of the $\mathrm{X}$-rays was $\lambda=1.033 \AA$. Data were recorded using a CCD-based detector (AVIEX) with a sample-detector distance of $3.13 \mathrm{~m}$ over a useful q-range $\left[0.004 \AA^{-1}, 0.25 \AA^{-1}\right]$, where $q=4 \pi \sin \theta / \lambda$ is the momentum transfer and $2 \theta$ is the scattering angle. All samples were prepared in $20 \mathrm{mM}$ Tris $\mathrm{HCl}$ buffer, $\mathrm{pH} 7.5,150 \mathrm{mM} \mathrm{NaCl}, 0.1$ $\mathrm{mM}$ EDTA, $2 \%$ glycerol, $10 \mathrm{mM} \mathrm{DTT}$, and $5 \mathrm{mM} \mathrm{MgCl}_{2}$ for $\mathrm{Mg}^{2+}$-containing samples. All measurements were performed at $10^{\circ} \mathrm{C}$. Data were recorded on ec-tmRNA with and without $\mathrm{Mg}^{2+}$ ions, alone and complexed with $\mathrm{SmpB}$ in a 1:1 ratio.

Samples were loaded on the Size Exclusion-High Performance Liquid Chromatography (SE-HPLC) column (Biosec 3 from Agilent Inc.) on-line with the SAXS measuring cell (David and Pérez 2009). Typically, $15 \mu \mathrm{L}$ of an $\sim 3 \mathrm{~g} / \mathrm{L}$ RNA solution were loaded on the column. Fifty successive frames of $1 \mathrm{sec}$ were recorded before the void volume and used as a background scattering pattern to be subtracted from the 250 frames recorded while the sample eluted from the column at a flow rate of $150 \mu \mathrm{L} / \mathrm{min}$. For each sample, SEC-SAXS measurements were complemented with standard measurements (batch mode) during which solutions were circulated continuously through the 1.5 -mm-diameter quartz capillary using the automatic sample changer (Agilent) at a flow-rate ensuring an irradiation time of $<1 \mathrm{sec}$. About 30 successive frames of $1 \mathrm{sec}$ each were recorded for RNA solutions and buffer alike, the concentration of RNA ranging from 0.25 to $\sim 3 \mathrm{mg} / \mathrm{mL}$.

\section{SAXS data analysis}

Normalization to the intensity of the transmitted beam and buffer subtraction was performed on-line for all scattering frames using the locally developed software FOXTROT. Individual patterns across the monomer peak were then examined using the program package PRIMUS (Petoukhov et al. 2012). The forward scattering $I(0)$ and the radius of gyration $\left(R_{\mathrm{g}}\right)$ were evaluated using the
Guinier approximation (Guinier 1939) $\ln [I(q)]=\ln [I(0)]-R_{\mathrm{g}}^{2} / 3$ $q^{2}$. Identical frames were averaged and the resulting curve spliced with batch data at larger angles for further analysis. The distance distribution function $p(r)$ was determined using the Indirect Fourier Transform approach as implemented in the program Gnom (Svergun 1992). $p(r)$ is the histogram of distances between any pair of elementary scattering volumes within the particle. Finally, an estimate of the hydrated molecular volume is obtained from the Porod expression:

$$
V=\frac{2 \pi^{2} I(0)}{\int_{0}^{\infty} q^{2}(I(q)-B) d q},
$$

where $B$ is a constant subtracted to account for the contribution of inhomogeneities within the particle.

\section{Molecular modeling of tmRNA and complex}

A cryo-electronmicroscopy study recently led to a low-resolution model of [tmRNA.SmpB] complex bound to stalled ribosomes (Ramrath et al. 2012). Using the available atomic structure of the TLD-SmpB module (Gutmann et al. 2003) together with models of major tmRNA regions based on known homologous RNA-motifs, the authors proposed a pseudoatomic model of the whole tmRNA complexed to SmpB. This structure was divided into eight modules as summarized in Table 2. Atomic coordinates of the MLD, which are lacking in the 3J18 pdb file, were reconstructed using ModeRNA (Rother et al. 2011). All modules were superimposed with their centers of mass at the origin so as to avoid any bias toward an initial starting model. Their relative positions and orientations were then randomly modified in a protocol that aimed at bringing the scattering pattern of the ensemble of all modules to fit the target experimental pattern. This rigid-body approach using a simulated annealing protocol is implemented in the program SASREF (Petoukhov and Svergun 2005). Distance restraints were imposed between $5^{\prime}$ and $3^{\prime}$ extremities of adjacent modules so as to make possible the RNA connectivity restoration after rigid body refinement. To that end, a fixed core was defined within each module leaving on each side a potentially flexible linker (from 1 to $5 \mathrm{nt}$ in length). The distance restraint between modules $i$ and $i+1$ was $3.5 \mathrm{~N}_{\mathrm{i}} \AA$, where $\mathrm{N}_{\mathrm{i}}$ is the sum of nucleotides in the $3^{\prime}$ linker of module $i$ and in the $5^{\prime}$ linker of module $i+1$. The phosphate-sugar backbone was resealed between adjacent modules using ModeRNA and its extended fragment library. Although connectivity restoration somewhat alters the scattering pattern of the model, its effect is limited and does not question the validity of the rigid body refinement step as shown in Figure 6B.

In all four cases (with/without $\mathrm{SmpB}$, with/without $\mathrm{Mg}^{2+}$ ), models are found in which the H2-TLD "arm" is found on either side of the loop. This is no surprise, as the X-ray scattering pattern is spherically averaged through thermal motion so that only distance, but no orientation information, is accessible. At low resolution, the two models look like mirror images of one another that have the same $p(r)$ function, hence the same scattering pattern. The choice of the model "hand" is thus based on external information-here, the electron microscopy model. For each scattering pattern, we selected models with the correct "hand," ending with a number of models ranging between 100 and 200, enough to provide a basis for further statistical analysis.

The program Alpraxin from the ATSAS suite (Konarev et al. 2006) orients the molecule in such a way that its principal inertia 
vectors are aligned with the coordinate axes. We applied it to the loop domains of all models (i.e., excluding domain $\mathrm{H} 2$ and the TLD) so that, afterward, the $Z$ coordinate of any point is a direct measure of its distance to the "mid-plane" of the loop defined by the first two inertia axes of the loop.

To determine the orientation of the TLD arm with respect to the loop, we considered two parameters determined with respect to the mid-plane of the loop. First, $\alpha$ is the angle between the vector linking the center of mass of the loop $\mathrm{O}$ and the projection on the mid-plane $\mathrm{M}$ of the hinge point of $\mathrm{H} 2$ domain with the loop (phosphorus atom 304) and the vector MP, where $\mathrm{P}$ is the projection on the mid-plane of phosphorus atom 363 at the far end of the TLD (see diagram in Supplemental Fig. 4, panel A3). Second, $Z$ is the $Z$-coordinate of phosphorus 363 that reports about the elevation of the TLD.

The analysis of the distribution of parameter values makes the implicit assumption that our sampling of the solution space is exhaustive. The distributions derived from all 300 models are practically identical to those obtained using the selected models with the correct "hand." This strongly suggests that our sampling is satisfactory, thereby validating our conclusions.

To create a model for the ec-tmRNA in the accommodation stage, 3iyq (T. thermophilus) structure was used in which each module of the E. coli model was superimposed onto the corresponding T. thermophilus module before substitution (Weis et al. 2010).

Finally, a sketch of the tt-tmRNA conformation in the pre-accommodation stage has been proposed by Kaur et al. (2006). It comprises only phosphorus atoms of RNA moiety together with SmpB $\mathrm{C} \alpha$ atoms (2ob7). We proceeded in a similar way to obtain a model for the ec-tmRNA in the pre-accommodation stage. Regarding the complex with $\mathrm{SmpB}$, the protein was added to module TLD+H2 in agreement with the crystallographic structure of the [TLD·SmpB] complex (Bessho et al. 2007).

\section{SUPPLEMENTAL MATERIAL}

Supplemental material is available for this article.

\section{ACKNOWLEDGMENTS}

We thank Pierre Roblin (INRA, Nantes, and SWING) for help with the SAXS measurements on the SWING beamline (proposals 20100133 and 20110109, SOLEIL synchrotron, Gif-sur-Yvette, France). This work was supported by grant (ANR-09-MIEN-03001) from the Agence Nationale pour la Recherche to S.N.-L. and F.D., as well as from the CNRS and from the French Department of Research and Education. E.R.-S. was a recipient of a fellowship from the Iranian government.

Received April 3, 2014; accepted June 15, 2014.

\section{REFERENCES}

Baik S, Inoue K, Ouyang M, Inouye M. 2009. Significant bias against the ACA triplet in the tmRNA sequence of Escherichia coli K-12. J Bacteriol 191: 6157-6166.

Barends S, Karzai AW, Sauer RT, Wower J, Kraal B. 2001. Simultaneous and functional binding of SmpB and EF-Tu-GTP to the alanyl acceptor arm of tmRNA. J Mol Biol 314: 9-21.

Bessho Y, Shibata R, Sekine S, Murayama K, Higashijima K, HoriTakemoto C, Shirouzu M, Kuramitsu S, Yokoyama S. 2007.
Structural basis for functional mimicry of long-variable-arm tRNA by transfer-messenger RNA. Proc Natl Acad Sci 104: 8293-8298.

Burks J, Zwieb C, Muller F, Wower I, Wower J. 2005. Comparative 3-D modeling of tmRNA. BMC Mol Biol 6: 14 .

Cheng K, Ivanova N, Scheres SH, Pavlov MY, Carazo JM, Hebert H, Ehrenberg M, Lindahl M. 2010. tmRNA.SmpB complex mimics native aminoacyl-tRNAs in the A site of stalled ribosomes. J Struct Biol 169: 342-348.

David G, Pérez J. 2009. Combined sampler robot and high-performance liquid chromatography: a fully automated system for biological small-angle X-ray scattering experiments at the Synchrotron SOLEIL SWING beamline. J Appl Crystallogr 42: 892-900.

Deckert G, Warren PV, Gaasterland T, Young WG, Lenox AL, Graham DE, Overbeek R, Snead MA, Keller M, Aujay M, et al. 1998. The complete genome of the hyperthermophilic bacterium Aquifex aeolicus. Nature 392: 353-358.

Dong G, Nowakowski J, Hoffman DW. 2002. Structure of small protein B: the protein component of the tmRNA-SmpB system for ribosome rescue. EMBO J 21: 1845-1854.

Fahrat MD, Rueda D. 2012. Fluorescence characterization of the tRNAlike domain of tmRNA in complex with SmpB. Biochemistry 51: 3531-3538.

Felden B, Gillet R. 2011. SmpB as the handyman of tmRNA during trans-translation. RNA Biol 8: 440-449.

Felden B, Himeno H, Muto A, McCutcheon JP, Atkins JF, Gesteland RF. 1997. Probing the structure of the Escherichia coli 10Sa RNA (tmRNA). RNA 3: 89-103.

Felden B, Hanawa K, Atkins JF, Himeno H, Muto A, Gesteland RF, McCloskey JA, Crain PF. 1998. Presence and location of modified nucleotides in Escherichia coli tmRNA: structural mimicry with tRNA acceptor branches. EMBO J 17: 3188-3196.

Fu J, Hashem Y, Wower I, Lei J, Liao HY, Zwieb C, Wower J, Frank J. 2010. Visualizing the transfer-messenger RNA as the ribosome resumes translation. EMBO J 29: 3819-3825.

Gaudin C, Nonin-Lecomte S, Tisne C, Corvaisier S, Bordeau V, Dardel F, Felden B. 2003. The tRNA-like domains of E. coli and A. aeolicus transfer-messenger RNA: structural and functional studies. J Mol Biol 331: 457-471.

Gillet R, Kaur S, Li W, Hallier M, Felden B, Frank J. 2007. Scaffolding as an organizing principle in trans-translation. The roles of small protein B and ribosomal protein S1. J Biol Chem 282: 6356-6363.

Guéron M, Plateau P, Descorps M. 1991. Solvent signal suppression in NMR. Prog Nucl Magn Reson Spectrosc 23: 135-209.

Guinier A. 1939. Diffraction of X-rays of very small angles - application to the study of ultramicroscopic phenomenon. Ann Phys 12: 161-237.

Gutmann S, Haebel PW, Metzinger L, Sutter M, Felden B, Ban N. 2003. Crystal structure of the transfer-RNA domain of transfer-messenger RNA in complex with SmpB. Nature 424: 699-703.

Hallier M, Ivanova N, Rametti A, Pavlov M, Ehrenberg M, Felden B. 2004. Pre-binding of small protein B to a stalled ribosome triggers trans-translation. J Biol Chem 279: 25978-25985.

Hallier M, Desreac J, Felden B. 2006. Small protein B interacts with the large and the small subunits of a stalled ribosome during trans-translation. Nucleic Acids Res 34: 1935-1943.

Hanawa-Suetsugu K, Takagi M, Inokuchi H, Himeno H, Muto A. 2002. SmpB functions in various steps of trans-translation. Nucleic Acids Res 30: 1620-1629.

Hong SJ, Tran QA, Keiler KC. 2005. Cell cycle-regulated degradation of tmRNA is controlled by RNase R and SmpB. Mol Microbiol 57: $565-575$.

Ito K, Tadaki T, Lee S, Takada K, Muto A, Himeno H. 2002. Transtranslation mediated by Bacillus subtilis tmRNA. FEBS Lett 516: 245-252.

Ivanov PV, Zvereva MI, Shpanchenko OV, Dontsova OA, Bogdanov AA, Aglyamova GV, Lim VI, Teraoka Y, Nierhaus KH. 2002. How does tmRNA move through the ribosome? FEBS Lett 514: 55-59.

Jacob Y, Sharkady SM, Bhardwaj K, Sanda A, Williams KP. 2005. Function of the SmpB tail in transfer-messenger RNA translation revealed by a nucleus-encoded form. J Biol Chem 280: 5503-5509. 
Kaur S, Gillet R, Li W, Gursky R, Frank J. 2006. Cryo-EM visualization of transfer messenger RNA with two SmpBs in a stalled ribosome. Proc Natl Acad Sci 103: 16484-16489.

Komine Y, Kitabatake M, Yokogawa T, Nishikawa K, Inokuchi H. 1994. A tRNA-like structure is present in 10Sa RNA, a small stable RNA from Escherichia coli. Proc Natl Acad Sci 91: 9223-9227.

Konarev PV, Petoukhov MV, Volkov VV, Svergun DI. 2006. ATSAS 2.1, a program package for small-angle scattering data analysis. J Appl Crystallogr 39: 277-286.

Konno T, Kurita D, Takada K, Muto A, Himeno H. 2007. A functional interaction of $\mathrm{SmpB}$ with tmRNA for determination of the resuming point of trans-translation. RNA 13: 1723-1731.

Lian W, Deutscher MP. 2012. Transfer-messenger RNA-SmpB protein regulates ribonuclease $\mathrm{R}$ turnover by promoting binding of HslUV and Lon proteases. J Biol Chem 287: 33472-33479.

Liu M, Mao X, Ye C, Huang H, Nicholson JK, Lindon JC. 1998. Improved WATERGATE pulse sequences for solvent suppression in NMR spectroscopy. J Magn Reson 132: 125-129.

Meinnel T, Mechulam Y, Fayat G. 1998. Fast purification of a functional elongator tRNAmet expressed from a synthetic gene in vivo. Nucleic Acids Res 16: 8095-8096.

Metzinger L, Hallier M, Felden B. 2005. Independent binding sites of small protein B onto transfer-messenger RNA during trans-translation. Nucleic Acids Res 33: 2384-2394.

Metzinger L, Hallier M, Felden B. 2008. The highest affinity binding site of small protein B on transfer messenger RNA is outside the tRNA domain. RNA 14: 1761-1772.

Neubauer C, Gillet R, Kelley AC, Ramakrishnan V. 2012. Decoding in the absence of a codon by tmRNA and SmpB in the ribosome. Science 335: 1366-1369.

Nonin-Lecomte S, Felden B, Dardel F. 2006. NMR structure of the Aquifex aeolicus tmRNA pseudoknot PK1: new insights into the recoding event of the ribosomal trans-translation. Nucleic Acids Res 34: $1847-1853$.

Nonin-Lecomte S, Germain-Amiot N, Gillet R, Hallier M, Ponchon L, Dardel F, Felden B. 2009. Ribosome hijacking: a role for small protein B during trans-translation. EMBO Rep 10: 160-165.

Petoukhov MV, Svergun DI. 2005. Global rigid body modeling of macromolecular complexes against small-angle scattering data. Biophys $J$ 89: $1237-1250$.

Petoukhov MV, Franke D, Shkumatov AV, Tria G, Kikhney AG, Gajda M, Gorba C, Mertens HDT, Konarev PV, Svergun DI. 2012. New developments in the ATSAS package for small-angle scattering data analysis. J Appl Crystallogr 45: 342-350.

Ponchon L, Beauvais G, Nonin-Lecomte S, Dardel F. 2009. A generic protocol for the expression and purification of recombinant RNA in Escherichia coli using a tRNA scaffold. Nat Protoc 4: 947-959.

Ponchon L, Catala M, Seijo B, El Khouri M, Dardel F, Nonin-Lecomte S, Tisné C. 2013. Co-expression of RNA-protein complexes in
Escherichia coli and applications to RNA biology. Nucleic Acids Res 41: e150.

Ramrath DJ, Yamamoto H, Rother K, Wittek D, Pech M, Mielke T, Loerke J, Scheerer P, Ivanov P, Teraoka Y, et al. 2012. The complex of tmRNA-SmpB and EF-G on translocating ribosomes. Nature 485: 526-529.

Ranaei-Siadat E, Fabret C, Seijo B, Dardel F, Grosjean H, NoninLecomte S. 2013. RNA-methyltransferase TrmA is a dual-specific enzyme responsible for C5-methylation of uridine in both tmRNA and tRNA. RNA Biol 10: 572-578.

Rother M, Rother K, Puton T, Bujnicki JM. 2011. RNA tertiary structure prediction with ModeRNA. Brief Bioinform 12: 601-613.

Shimizu Y, Ueda T. 2002. The role of SmpB protein in trans-translation. FEBS Lett 514: 74-77.

Someya T, Nameki N, Hosoi H, Suzuki S, Hatanaka H, Fujii M, Terada T, Shirouzu M, Inoue Y, Shibata T, et al. 2003. Solution structure of a tmRNA-binding protein, $\mathrm{SmpB}$, from Thermus thermophilus. FEBS Lett 535: 94-100.

Sundermeier TR, Karzai AW. 2007. Functional SmpB-ribosome interactions require tmRNA. J Biol Chem 282: 34779-34786.

Sundermeier TR, Dulebohn DP, Cho HJ, Karzai AW. 2005. A previously uncharacterized role for small protein $\mathrm{B}(\mathrm{SmpB})$ in transfer messenger RNA-mediated trans-translation. Proc Natl Acad Sci 102: 2316-2321.

Svergun DI. 1992. Determination of the regularization parameter in indirect-transform methods using perceptual criteria. J Appl Crystallogr 25: 495-503.

Tanner DR, Dewey JD, Miller MR, Buskirk AR. 2006. Genetic analysis of the structure and function of transfer messenger RNA pseudoknot 1. J Biol Chem 281: 10561-10566.

Thibonnier M, Aubert S, Ecobichon C, De Reuse H. 2010. Study of the functionality of the Helicobacter pylori trans-translation components SmpB and SsrA in an heterologous system. BMC Microbiol 10: 91.

Valle M, Gillet R, Kaur S, Henne A, Ramakrishnan V, Frank J. 2003. Visualizing tmRNA entry into a stalled ribosome. Science 300: 127-130.

Weis F, Bron P, Giudice E, Rolland JP, Thomas D, Felden B, Gillet R. 2010. tmRNA-SmpB: a journey to the centre of the bacterial ribosome. EMBO J 29: 3810-3818.

Wower IK, Zwieb CW, Guven SA, Wower J. 2000. Binding and crosslinking of tmRNA to ribosomal protein S1, on and off the Escherichia coli ribosome. EMBO J 19: 6612-6621.

Wower J, Zwieb CW, Hoffman DW, Wower IK. 2002. SmpB: a protein that binds to double-stranded segments in tmRNA and tRNA. Biochemistry 4: 8826-8836.

Zhang Y, Zhang J, Hoeflich KP, Ikura M, Qing G, Inouye M. 2003. MazF cleaves cellular mRNAs specifically at ACA to block protein synthesis in Escherichia coli. Mol Cell 12: 913-923.

Zuker M. 2003. Mfold web server for nucleic acid folding and hybridization prediction. Nucleic Acids Res 31: 3406-3415. 
RNA 20: $1607-1620(2014)$

\section{In vivo tmRNA protection by SmpB and pre-ribosome binding conformation in solution}

EHSAN RANAEI-SIADAT, CÉCILE MÉRIGOUX, BILI SEIJO, LUC PONCHON, JEAN-MICHEL SALIOU, JULIE BERNAUER, SARAH SANGLIER-CIANFÉRANI, FRÉDERIC DARDEL, PATRICE VACHETTE, and SYLVIE NONIN-LECOMTE

In the above-noted article, on p. 1607, the fifth affiliation, "CNRS, IPHC-LSMBO, Université Louis Pasteur Bât, 67087 Strasbourg, France," should be replaced by "Laboratoire de Spectrométrie de Masse BioOrganique (LSMBO), IPHC, Université de Strasbourg, CNRS, UMR7178, 67087 Strasbourg, France.”

The authors apologize for this error. 

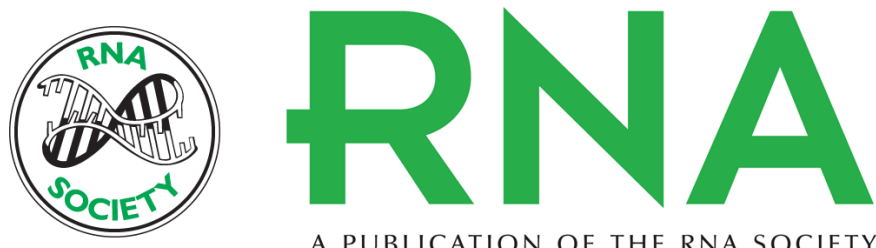

A PUBLICATION OF THE RNA SOCIETY

\title{
In vivo tmRNA protection by SmpB and pre-ribosome binding conformation in solution
}

\author{
Ehsan Ranaei-Siadat, Cécile Mérigoux, Bili Seijo, et al.
}

RNA 2014 20: 1607-1620 originally published online August 18, 2014

Access the most recent version at doi:10.1261/rna.045674.114

\section{Supplemental http://rnajournal.cshlp.org/content/suppl/2014/08/05/rna.045674.114.DC1 Material}

Related Content

References

Creative

Commons

License

Email Alerting
Service
In vivo tmRNA protection by SmpB and pre-ribosome binding conformation in solution

Ehsan Ranaei-Siadat, Cécile Mérigoux, Bili Seijo, et al.

RNA November , 2014 20: 1827

This article cites 57 articles, 22 of which can be accessed free at:

http://rnajournal.cshlp.org/content/20/10/1607.full.html\#ref-list-1

Articles cited in:

http://rnajournal.cshlp.org/content/20/10/1607.full.html\#related-urls

This article is distributed exclusively by the RNA Society for the first 12 months after the full-issue publication date (see http://rnajournal.cshlp.org/site/misc/terms.xhtml). After 12 months, it is available under a Creative Commons License (Attribution-NonCommercial 4.0 International), as described at http://creativecommons.org/licenses/by-nc/4.0/.

Receive free email alerts when new articles cite this article - sign up in the box at the top right corner of the article or click here. 